\title{
Improving the diagnosis of pulmonary embolism in the emergency department
}

\author{
Jenni Cooper \\ Western Health, Melbourne, Australia
}

\begin{abstract}
The diagnosis of pulmonary embolism (PE) in the emergency department is challenging due to the wide range of non-specific symptoms, lack of clinical diagnostic criteria, and imperfect investigations. Various scoring systems exist in an attempt to limit unnecessary investigations in those with low risk of PE. Following a baseline audit and subsequent PDSA cycles we implemented a flowchart for use in patients suspected of pulmonary embolism encouraging the correct use of the Wells Score and Pulmonary Embolism Rule out Criteria (PERC).

The standard used for comparison was based on the NICE guidelines for diagnosis of PE with the addition that PERC could also be used if appropriate. Data was collected over four week periods before and after the introduction of our flowchart in two emergency departments in Melbourne. We aimed to increase documentation of pre-test probability, reduce inappropriate investigations, and increase the use of interim parenteral anticoagulation where there was a delay to imaging.
\end{abstract}

Results showed an increase in the documentation of pre-test probability and the proportion of investigations requested that were inappropriate was reduced. The percentage of inappropriate d-dimers was reduced from $36 \%$ to $24 \%$; the percentage of inappropriate CTPAs was reduced from $34 \%$ to $10 \%$; and the percentage of inappropriate V/Q scans was reduced from $42 \%$ to $14 \%$.

Implementation of a simple diagnostic algorithm led to an increase in documentation of pre-test probability and a reduction in inappropriate and unnecessary investigations. This intervention may be applicable to other emergency departments where similar issues in diagnosing pulmonary embolism exist.

\section{Problem}

Prior to undertaking this project it was felt that, as a department, we may request a significant number of unnecessary tests that could potentially led to significant adverse effects such as contrastinduced nephropathy, contrast allergy or anaphylaxis, and unnecessary radiation exposure.

The PERC is commonly accepted for use in patients with a low pretest probability of $\mathrm{PE}$, allowing the diagnosis to be excluded without requiring d-dimer testing (hence avoiding some inevitable false positive d-dimers) although it was unclear if this was being widely used. It was also suspected that patients awaiting imaging for investigation of suspected PE were not always treated with interim anticoagulation which the NICE guidelines would recommend; this had been raised as a risk management issue following a clinical incident.

\section{Background}

The diagnosis of pulmonary embolism (PE) in the emergency department is challenging due to the lack of clinical diagnostic criteria and imperfect investigations. The presenting symptoms are common and non-specific varying from chest pain, shortness of breath, cough and haemoptysis, to syncope and cardiac arrest.[1] There is a high false positive rate with non-invasive testing $(d-$ dimer) but relatively significant potential adverse events (radiation and contrast associated complications) with more invasive testing such as Computed Tomography Pulmonary Angiogram (CTPA).[2]

Various scoring systems exist in an attempt to limit unnecessary imaging for those identified as having a low pre-test probability of pulmonary embolism without increasing the risk of missing the potentially life threatening diagnosis of pulmonary embolism.[3] Using a combination of the Wells score and either a d-dimer (if PERC positive) or no testing (if PERC negative) can reduce the need for imaging.[4-6] These scoring systems are particularly useful for junior doctors who may lack clinical experience. The Pulmonary Embolism Rule-Out Criteria (PERC) has been validated for use within the emergency department.[7] Within the emergency departments in which this project was undertaken there were no specific guidelines that junior doctors were required to follow although discussion with senior clinicians prior to requesting a ddimer or imaging was encouraged.

The National Institute for Health and Care Excellence (NICE) guidelines describe proposed best standards that should be met when considering the diagnosis of pulmonary embolism which involves appropriate use of the Wells scoring system.[8]

Below is a summary of these guidelines:

Patients with signs or symptoms of pulmonary embolism should all 
BMJ Quality Improvement Reports

have an assessment of their general medical history, a physical examination, and a chest X-ray. All patients with suspected pulmonary embolism should have their probability of pulmonary embolism assessed using the two-level Pulmonary Embolism Wells score.

Patients with a likely two-level Wells score should NOT have a ddimer and be offered CTPA with the exception of patients with contrast allergy, renal impairment, or in whom risk of irradiation is high who should instead be offered a Ventilation Perfusion (V/Q) scan. All patients not able to have immediate imaging should have interim parenteral anticoagulant therapy.

Patients with an unlikely two-level Wells score should all have a ddimer test and no patients offered CTPA until d-dimer result available. All patients with positive d-dimer are offered CTPA with the exception of patients with contrast allergy, renal impairment, or in whom risk of irradiation is high who should instead be offered a V/Q scan. All patients who cannot have a CTPA or V/Q carried out immediately should have interim parenteral anticoagulant therapy.

However, although not part of the NICE guidelines, in a select group of patients with an unlikely two-level Wells score (in keeping with the clinical gestalt of an experienced clinician) who fulfill all 8 PERC criteria a d-dimer is not necessarily indicated.[1,2]

These criteria are:

- Age $<50$

- Pulse $<100 \mathrm{bpm}$

- Saturations $\geq 95 \%$

- No haemoptysis

- No oestrogen use

- No surgery/trauma requiring hospitalization within 4 weeks

- No prior venous thromboembolism (VTE)

- No unilateral leg swelling

\section{Baseline measurement}

A baseline audit was designed to investigate the diagnosis of pulmonary embolism in the emergency department in which we compared current practice in two emergency departments within the hospital network to a set standard. The standard which was used was based on the NICE guideline on diagnosis of venous thromboembolism but with the addition that the Pulmonary Embolism Rule-out Criteria could also be used to differentiate low risk from no risk pulmonary embolism therefore avoiding unnecessary d-dimer use in very low risk patients.

Data was collected over a four week period and aimed to include all patients where the diagnosis of pulmonary embolism was considered. Search criteria, inclusion criteria, and exclusion criteria used were identical to those used in the subsequent PDSA cycles and re-audit. The baseline audit was carried out over a four week period in March 2015 and the re-audit carried out over a four week period in June 2015. electronic patient records by either diagnosis or presenting symptoms.

\section{Search Criteria:}

- Patients either admitted to a ward or the emergency observation unit with diagnosis of 'PE' or 'PE for exclusion'

- Patients attending the emergency department with symptoms that could be suggestive of pulmonary embolism (chest pain/shortness of breath/haemoptysis/cough)

Inclusion Criteria:

- Adults attending the emergency department who had either a d-dimer (where the consideration of PE was documented in the notes), a CTPA, or a V/Q scan requested.

- Patients presenting with dyspnoea, chest pain, haemptysis, or cough in whom a diagnosis of pulmonary embolism was considered.

\section{Exclusion Criteria:}

- Pregnant women

- Patients under 18

- Investigations requested outside of the emergency department (e.g. by GP/inpatient team)

The data pro forma that was used was designed to allow comparison of data collected to the NICE clinical guidelines (Venous thromboembolic disease - Diagnosis of Pulmonary Embolism) but including measurement of PERC score.

\section{See Diagnosis of PE Data Pro Forma}

The duration of data collection was chosen to allow sufficient numbers to be included so that changes could be identified more accurately.

110 patients were eligible and included in the audit. The results showed that there was $100 \%$ compliance with assessment of history, exam, and CXR as well as consideration of alternate diagnoses. However the initial audit findings did suggest several potential areas for improvement:

1 There was poor documentation of Wells score or clinical probability (pre-test probability according to clinical gestalt) - 79\% not documented.

2 There were inappropriate or unnecessary investigations requested that might have been avoided if the guidelines were followed or PERC rule utilised appropriately.

3 D-dimers: $36 \%$ of $d$-dimers that were sent were unnecessary or inappropriate

CTPAs: $34 \%$ of the CTPAs requested were unnecessary or inappropriate 
V/Q scans: $42 \%$ of the $V / Q$ scans requested were unnecessary or inappropriate

4 The majority of patients undergoing imaging for suspected PE did not receive interim anticoagulation $37.5 \%$ received anticoagulation).

See supplementary file: ds6615.docx - "Data Pro Forma"

\section{Design}

Following the baseline audit the aims of the quality improvement were specified:

1 To improve documentation of pre-test probability (Wells score/clinical gestalt of senior clinician) which should be documented in $100 \%$ of cases.

2 Investigations should be in line with pre-test probability which could subsequently:

a Reduce unnecessary d-dimers through appropriate use of PERC and Wells criteria.

b Reduce unnecessary imaging through appropriate use of $d$ dimers in patients with low pre-test probability of PE.

3 To increase use of interim anti-coagulation in patients undergoing CTPA or $V / Q$ where there is a delay to imaging.

The potential benefits may include a cost benefit from a reduction in unnecessary investigations and reduced risk to patients from radiation/potential complications from contrast (nephropathy/renal impairment).

The initial intervention considered was to design a flow chart poster for display in the relevant department areas encouraging clinicians to assess the pre-test probability of pulmonary embolism prior to requesting pathology or radiology and to remind clinicians requesting imaging with CTPA and $V / Q$ to first prescribe interim parenteral anti-coagulation. This would also be associated with an additional poster documenting how to calculate the Wells and PERC score for ease of use in the department.

See Flowchart

In comparison to having educational sessions alone this should be more sustainable as compliance should not be affected to the same degree when the junior staff change rotation. An associated education session or presentation introducing the flowchart was felt to be necessary to introduce the flow chart and encourage its use by explaining the potential advantages.

Other options for interventions were considered including introducing a version of the flowchart into the existing emergency observation units' 'Pulmonary Embolism for Exclusion' pathway to be completed as well as designing stickers with Wells scoring criteria printed onto them to be placed in the patient's notes with a tick box to encourage compliance. After surveying medical staff it was felt that given the existing time pressures in the emergency department having additional paperwork to complete would be an unpopular option and therefore poor compliance with these additional interventions would be likely.

\section{Strategy}

Several Plan, Do, Study, Act (PDSA) cycles were used during the development and implementation phase of the quality improvement project. Data was sampled from one emergency department over one week periods prior to and after each PDSA cycle to measure change.

PDSA cycle 1 - Following discussion with the heads of departments the baseline audit results and proposed flowchart were circulated to the consultant group for comment and approval.

This led to improvements in flowchart and permission was gained to continue with the planned intervention.

PDSA cycle 2 - The baseline audit results and proposed flowchart were presented to the Emergency Medicine registrar group to explain audit findings and introduce the flowchart.

This encouraged use of the scoring systems and feedback was gained which suggested that any interventions requiring extra paperwork would likely have poor compliance given time pressures.

PDSA cycle 3 - Introduction of flowchart into clinical areas.

During implementation of change using these PDSA cycles data was collected in one of the emergency departments over several one week periods. These varied in sample size from 6 to 17 due to the variation in numbers of patients meeting the eligibility criteria.

The percentage of investigations which were requested in line with the standard during the PDSA cycles were:

Baseline Measurement $1-67 \%$ (6 out of 9 )

Measurement $2-67 \%$ (4 out of 6 )

Measurement $3-100 \%$ (2 out of 2$)$

Measurement $4-67 \%$ (2 out of 3 )

Given the small sample size it was not clear whether significant improvements were being made. As planned a re-audit over the same time frame as the initial audit was carried out after introduction of the flowchart.

\section{Results}

The results from the re-audit showed an improvement in documentation of the Wells score from 19 out of $110(17 \%)$ to 23 out of $81(28 \%)$; and in documentation of pre-test probability 
according to clinical gestalt from 4 out of $110(4 \%)$ to 19 out of 81 $(23 \%)$. There was also a reduction in the number of inappropriate or unnecessary investigations being requested.

Unnecessary/inappropriate d-dimers consisted of those d-dimers performed in patients whose wells score indicated PE was likely (and therefore should have gone straight to imaging) and d-dimers that were requested patients with a low pre-test probability who would not have required a d-dimer if the PERC had been applied.

The percentage of total d-dimers sent that were inappropriate/unnecessary was $36 \%$ in the audit group and $24 \%$ in the re-audit group.

In the baseline audit the total number of inappropriate/unnecessary d-dimers was 24. 2 were sent in patients who had a high pre-test probability (Wells likely) so required imaging regardless of the result. 22 were sent in patients that had a low pre-test probability (Wells unlikely) and were PERC negative and so could have been avoided, and of these 4 were positive. 2 had subsequent negative CTPAs, 1 had a subsequent negative V/Q scan, and 1 was not further investigated. None had a final diagnosis of PE.

In the re-audit the total number of inappropriate/unnecessary ddimers was 12. 2 were sent in patients who were in the Wells likely category. 10 were sent in patients who had a Wells score of zero and would not have required a d-dimer if the PERC had been applied.

Unnecessary/inappropriate imaging with V/Q and CTPA consisted of those patients undergoing imaging who had an unlikely Wells score with the exception of those who had a positive d-dimer that was appropriately requested.

A total of 38 CTPAs were performed in the initial audit 25 of which were requested in line with the guidelines. 4 could have been avoided as they had a low pre-test probability and either met PERC criteria or had a negative d-dimer result. 9 were performed in patients with a low pre-test probability without an initial d-dimer, some of which could possibly have been avoided if $d$-dimer had been requested and returned negative.

In the re-audit 31 CTPAs were performed 28 of which were requested within the guidelines. 2 were requested in patients within the Wells unlikely category who either had a negative d-dimer or would have satisfied the PERC criteria. 1 further CTPA was requested in a patient within the Wells unlikely category who did not have a d-dimer sent and therefore this was potentially avoidable depending on the d-dimer result.

A total of 12 patients were investigated with V/Q scan over the initial audit period. 3 were unnecessarily requested in patients with low pre-test probability and negative PERC. These were all negative for PE. 1 more V/Q scan was unnecessarily requested in a patient who had a low pre-test probability of PE according to the Wells score, who was PERC positive but who had had a negative d-dimer result. This was negative for $P E$. A further $V / Q$ was requested in a patient who had a low pre-test probability for PE, PERC positive who did not have a d-dimer requested so this possibly could have been avoided if they had had a negative $d$-dimer. This V/Q was also negative for $P E$. The remaining 7 were appropriately requested. In the re-audit period $7 \mathrm{~V} / \mathrm{Q}$ scans were requested only one of which was requested outside the guidelines.

With regards to interim treatment with parenteral anticoagulation if there was a delay to imaging, the results of the re-audit showed a reduction in the percentage treated from $35 \%$ to $18 \%$.

Overall 12 of 110 were diagnosed with PE on CTPA or V/Q scan $(11 \%)$ in the audit group and 7 out of 81 in the re-audit group (9\%).

See supplementary file: ds6617.pdf - "Flowchart"

\section{Lessons and limitations}

Lessons

As expected there was an improvement in the documentation of the Wells score following the intervention however this was not as significant an improvement as anticipated. The main reason for this is thought to be time pressures within the busy emergency departments limiting the thoroughness of documentation.

There was an improvement in investigation with the correct use of Wells score and use of PERC where appropriate. This may have been due to an increased awareness of the need to request investigations in line with the pre-test probability following the intervention even where this was not specifically documented. Again there is still room for further improvement but it may also be the case that there were other reasons for proceeding straight to imaging even in patients with a low pre-test probability. For example CT may be expected to have revealed another cause for the pain or dyspnoea even if it did not show a pulmonary embolism. Another reason might be if a d-dimer was not anticipated to be helpful either because the patient was elderly (so it was expected to positive regardless of whether they had a PE) or if the patient's symptoms had been ongoing for a longer period of time (so there were concerns d-dimer may be false negative).

Finally, the use of interim parenteral anticoagulation where there was a delay to imaging actually decreased following the intervention. The reason for this may be that a delay to imaging was not considered significant enough to warrant empirical treatment as the NICE guidelines define a delay to imaging as anytime greater than one hour which is very a short period of time. After presentation of the baseline audit findings at an emergency department teaching session to registrars and consultants there was discussion regarding the lack of evidence for empirical treatment given that the incidence of PE in those undergoing CTPA was $21 \%$ and so four out of five patients would receive unnecessary anticoagulation. As both emergency departments had 24/7 access to CT imaging many felt that as there would be unlikely to be a significant delay to imaging and the onset of action of subcutaneous low molecular weight heparin is such that it may be more appropriate to wait for imaging, thus only treating those who have proven pulmonary embolism. 
BMJ Quality Improvement Reports

Limitations

The main limitation for this project was lack of documentation. Data was retrospectively collected from medical and nursing records uploaded onto the electronic medical record system. Not all criteria required for the Wells/PERC criteria were documented. For the purpose of calculating the Wells score and PERC in patients in which it was not explicitly documented in the notes, in the absence of documentation of symptoms/risk factors (e.g.

haemoptysis/previous history/recent surgery/immobilisation) these were presumed not to be present. This may have resulted in an overestimation of Wells unlikely and PERC negative patients.

It is likely that pre-test probability according to clinical gestalt was assessed more often than it was documented in the notes and it is possible this could have varied from Wells likelihood category. However, in the few patients who had the clinical likelihood documented it was in keeping with the pre-test probability as per Wells score as either documented or calculated retrospectively.

It is possible that the search criteria may have missed a small number of patients who had a diagnosis of PE considered as it either wasn't documented as a potential diagnosis and therefore not included or their presenting symptom may have not fallen within the search criteria of dyspnoea/chest pain/haemoptysis/cough. There were numerous $d$-dimers sent without documentation of the reason and it was not appropriate to presume this was definitely to look for $\mathrm{PE}$ as it is possible it may have been related to potential aortic dissection, so this may have led to exclusion of patients who had the diagnosis considered but not documented in the notes.

Time pressures within the emergency department may have influenced whether $\mathrm{d}$-dimers were sent prior to imaging as this can prolong the time spent in the department and lead to a requirement for a short stay admission. However it could be argued that time pressures should not affect whether patient should be subjected to the increased radiation/risk of nephropathy/allergy from unnecessary investigation with CTPA. Time pressures may also be the main contributing factor to lack of documentation of pre-test probability.

These limitations were recognised and in order to minimise the effect the methods, search criteria, inclusion criteria, and exclusion criteria were all kept consistent between the initial audit and re-audit group.

Overall the implementation of the flowchart and the educational session were successful at reducing the number of unnecessary and inappropriate investigations when considering a diagnosis PE. One of the main difficulties with introducing the flowchart was encouraging medical staff to use it. In an attempt to increase engagement with the flowchart it was planned to incorporate it into the department's existing 'Pulmonary Embolism for Exclusion Pathway' that is required to be completed for all patients admitted into the short stay unit whilst awaiting investigation results. This should improve compliance with the flowchart and make these positive changes more sustainable.

\section{Conclusion}

Implementation of a flowchart utilising a simple diagnostic algorithm led to an increase in documentation of pre-test probability and a reduction in inappropriate investigations. This has reduced unnecessary imaging which could reduce costs but perhaps more importantly could also reduce the incidence of adverse effects such a contrast allergy, nephropathy, and unnecessary radiation exposure. This was a cheap and sustainable intervention which allows easy reference within the clinical area and has not increased the requirement for paperwork completion in a time pressured environment. This intervention may be applicable to other emergency departments where similar issues in diagnosing pulmonary embolism exist.

\section{References}

1 Stein PD, Terrin ML, Hales CA, Palevsky HI, Saltzman HA, Thompson BT, et al. Clinical, laboratory, roentgenographic, and electrocardiographic findings in patients with acute pulmonary embolism and no pre-existing cardiac or pulmonary disease. Chest. 1991;100(3):598-603.

2 Perrier A. D-dimer for suspected pulmonary embolism: Whom should we test? Chest 2004;125:807-9.

3 http://www.nice.org.uk/guidance/cg144/evidence/cg144-venousthromboembolic-diseases-full-guideline3. Accessed July 2015.

4 Wells PS, Anderson DR, Rodger M, Ginsberg JS, Kearon C, Gent $\mathrm{M}$, et al. Derivation of a Simple Clinical Model to Categorize Patients Probability of Pulmonary Embolism: Increasing the Models Utility with the SimpliRED D-dimer. Thromb Haemost.

2000;83(3):416-20.

5 Kline JA, Mitchell AM, Kabhrel C, Richman PB, Courtney DM. Clinical criteria to prevent unnecessary diagnostic testing in emergency department patients with suspected pulmonary embolism. J Thromb Haemost. 2004;2(8):1247-55.

6 Carpenter CR, Keim SM, Seupaul RA, Pines JM; Best Evidence in Emergency Medicine Investigator Group. Differentiating Low Risk and No Risk PE Patients: the PERC score. J Emerg Med. 2009;36(3):317-22.

7 Singh B, Mommer SK, Erwin PJ, Mascarenhas SS, Parsaik AK. Pulmonary embolism rule-out criteria (PERC) in pulmonary embolism--revisited: a systematic review and meta-analysis. Emerg Med J. 2013 Sep;30(9):701-6. doi: 10.1136/emermed-2012-201730. Epub 2012 Oct 4.

8 National Institute for Health and Care Excellence 2012 Venous thromboembolic diseases: the management of venous thromboembolic diseases and the role of thrombophilia testing. https://www.nice.org.uk/guidance/cg144/chapter/1-Guidance\#diagn osis-2 accessed July 2015. 
9 http://www.nice.org.uk/guidance/cg144/evidence/cg144-venousthromboembolic-diseases-appendix-c2. Accessed July 2015.

\section{Declaration of interests}

Nothing to Declare

\section{Acknowledgements}

Dr Andrew Tagg, Dr Gary Ayton

\section{Ethical approval}

Approval for the project was gained from the local low risk human research ethics panel. 